\title{
Anemia en el primer control de gestantes en un centro de salud de Lima, Perú y su relación con el estado nutricional pregestacional
}

\author{
Blanca Regina Taipe-Ruiz* 1, a ; Luzmila Troncoso-Corzo 2,b
}

RESUMEN

Objetivo: Determinar la frecuencia de anemia en gestantes y su relación con el estado nutricional pregestacional. Materiales y métodos: Estudio descriptivo, observacional, transversal y retrospectivo, realizado en el Servicio de Obstetricia y Nutrición del Centro de Salud del distrito de Santiago de Surco entre el 1 de julio de 2015 y el 31 julio de 2016. Se revisaron 455 historias clínicas materno-perinatales (HCMP) del servicio de Obstetricia del Centro de Salud de Surco de las que se seleccionaron 93 que cumplian los criterios de inclusión. Se utilizó estadística descriptiva.

Resultados: El 11,8 \% de gestantes presentaron anemia. La hemoglobina promedio en las gestantes fue 12,2 $\pm 1,06 \mathrm{~g} / \mathrm{dl}$. El $58,5 \%$ tenía índice de masa corporal (IMC) pregestacional normal y también una mayor frecuencia de anemia (10,7 \%). Se observó que los casos de anemia son más frecuentes en los dos primeros trimestres, con un promedio de 4,8 \%, y disminuyen en el tercer trimestre $(2,2 \%)$.

Conclusiones: La mayor frecuencia de anemia en mujeres embarazadas se presentó en el grupo con IMC pregestacional normal. Los niveles de hemoglobina disminuyen de acuerdo a la edad de la madre y aumentan conforme a la edad gestacional.

Palabras clave: Anemia; Deficiencia de hierro; Estado nutricional; Atención prenatal (Fuente: DeCS BIREME).

\section{Anemia at the first prenatal visit in a health center in Lima, Peru, and its relationship with the pregestational nutritional status}

\section{ABSTRACT}

Objective: To determine the frequency of anemia in pregnant women and its relationship with the pregestational nutritional status.

Materials and methods: A descriptive, observational, cross-sectional and retrospective study was carried out in the Obstetrics and Nutrition Department of the Centro de Salud de Surco between July 1, 2015 and July 31, 2016. Four hundred fifty-five (455) maternal perinatal medical records (HCMP) from the Obstetrics Department of the Centro de Salud de Surco were reviewed, out of which 93 met the inclusion criteria and were selected. Descriptive statistics was used.

Results: Eleven point eight percent $(11.8 \%)$ of the pregnant women presented anemia. The average hemoglobin level in the aforementioned women was $12.2 \pm 1.06 \mathrm{~g} / \mathrm{dl}$. Fifty-eight point five percent $(58.5 \%)$ had a normal pregestational body mass index (BMI) and the highest frequency of anemia (10.7\%). It was observed that cases of anemia were more frequent in the first two quarters of pregnancy, reaching on average $4.8 \%$, and decreased in the third quarter (2.2\%).

Conclusions: The highest frequency of anemia in pregnant women occurred in the normal pregestational BMI group. Hemoglobin levels decrease according to the age of the mother and increase according to the gestational age.

Keywords: Anemia; Iron deficiency; Nutritional status; Prenatal care (Source: MeSH NLM).

1. Centro de Salud de Surco. Lima, Perú.

2. Universidad Nacional Mayor de San Marcos, Facultad de Medicina. Lima, Perú.

a. Maestría en Nutrición Clínica, Licenciada en Nutrición.

b. Doctora en Medicina, Médico Cirujano, Docente investigador.

* Autor corresponsal. 


\section{INTRODUCCIÓN}

En todo el mundo, el déficit de hierro es la causa más frecuente de anemia en el embarazo. La anemia es la reducción del número normal de eritrocitos circulantes y de la cantidad de hemoglobina en la sangre. Más de medio millón de muertes maternas ocurren cada año y, aproximadamente, el $90 \%$ de las mismas en los países en desarrollo ${ }^{(1)}$.

La prevalencia de gestantes anémicas a nivel mundial, se ha logrado reducir de $43 \%$ en 1995, a $38 \%$ en 2011; y de $37 \%$ a $31 \%$ en países de América Latina y el Caribe en el mismo tiempo. El Perú logró una reducción de $42,7 \%$ en el $2004{ }^{(2)}$, a 24,8 \% al primer semestre $2014{ }^{(3)}$. Sin embargo, las regiones de Huancavelica y Puno se encuentran con una prevalencia mucho mayor a nivel nacional de $46,8 \%{ }^{(4)}$. A pesar de esta reducción, la anemia en el periodo gestacional a nivel nacional persiste como un problema de Salud Pública ${ }^{(2)}$.

La Encuesta Nacional de Demografía y Salud (ENDES) señala que la prevalencia de gestantes anémicas es ocasionada por la ingesta inadecuada de hierro, que se encuentra por debajo del requerimiento nutricional de la gestante (27 mg/día) ${ }^{(2,4)}$.

El estado nutricional de la mujer, antes y durante el embarazo, es un factor fundamental para la salud de ella misma y la de su hijo. Es una situación importante que debe ser considerada, ya que estas mujeres constituyen un grupo vulnerable desde el punto de vista nutricional, especialmente en los países en desarrollo ${ }^{(5)}$.

La evaluación del estado nutricional en el proceso gestacional, según medidas antropométricas, es fundamental ya que permite conocer riesgos $\mathrm{y}$, a la vez, complicaciones futuras en la madre y el feto. Con el diagnóstico nutricional es posible establecer estrategias de recuperación o mantenimiento de la salud, lo que contribuye al bienestar y calidad de vida de las gestantes ${ }^{(6)}$.

La anemia está asociada al incremento de la morbilidad y mortalidad de grupos poblacionales vulnerables, en particular, las embarazadas y los niños pequeños. La transferencia de bajas concentraciones de hierro de la madre al feto podría conducir a situaciones como parto pretérmino, bajo peso al nacer, el tamaño pequeño para la edad y morbimortalidad infantil (7).

La reserva de hierro presente en la mujer adulta es escasa y suele variar entre 100 y $700 \mathrm{mg}$. La gestante necesita unos $600 \mathrm{mg}$ de hierro para formar su propia sangre y unos $375 \mathrm{mg}$ para transferir al feto. La cantidad de hierro absorbido en la dieta junto con el extraído de las reservas maternas suele ser insuficiente para suplir esas demandas, de tal manera que, con mucha frecuencia, se desarrolla anemia en el embarazo. Si además de eso se consideran los $200 \mathrm{mg}$ de hierro que se pierden en el sangrado del alumbramiento y puerperio, y la transferencia de hierro en la leche materna $(1,1 \mathrm{mg}$ de hierro/litro de leche), se plantea un problema cuya prevención debe empezar antes del embarazo, mediante la mejora de las reservas de hierro de la mujer en edad fértil (8).

Se presume que la deficiencia en la reserva de hierro en el organismo conduce a una deficiente eritropoyesis y a un balance negativo de hierro, justamente cuando el suministro del mismo en la dieta es insuficiente para mantener la concentración normal de hemoglobina $(\mathrm{Hb})$. Sin embargo, la concentración de hemoglobina se mantiene normal con una ingesta de hierro del $80 \%$ de la dosis recomendada en la primera mitad de la gestación y de $41 \%$, en la segunda mitad ${ }^{(9)}$.

El objetivo del trabajo fue determinar la frecuencia de anemia en gestantes y su relación con el estado nutricional pregestacional.

\section{MATERIALES Y MÉTODOS}

Diseño y población

Estudio descriptivo, observacional, transversal y retrospectivo, en el que se revisaron $\mathbf{4 5 5}$ historias clínicas maternas perinatales (HCMP) del Servicio de Obstetricia y Nutrición del Centro de Salud de Surco donde se realiza atención de primer nivel en el periodo comprendido entre 1 de julio del 2015 a 31 de julio del 2016. Se seleccionaron 93 HCMP que cumplían con los criterios de inclusión (variables completas). De acuerdo a lo que establece la Norma Técnica de Salud para la Atención Integral de Salud Materna ${ }^{(10)}$, todas las gestantes son derivadas al Servicio de Nutrición para realizar la evaluación nutricional.

\section{Variables y mediciones}

Las mediciones antropométricas (peso y talla) se realizaron de acuerdo a las recomendaciones del Manual de la Medición del Peso y Talla del Ministerio de SaludInstituto Nacional de Salud. Para asegurar datos exactos en la medición, se utilizó una balanza de precisión con capacidad de $150 \mathrm{~kg}$ y precisión de $0,1 \mathrm{~kg}$ y un tallímetro de madera elaborado según especificaciones técnicas del Centro Nacional de Alimentación y Nutrición (CENAN) (11). La hemoglobina se determinó por medición de hematocrito por microcentrifugación y después se dividió el valor entre tres, se consideró la primera medición de hemoglobina durante el periodo de gestación.

El estado nutricional pregestacional se definió con el indicador de índice de masa corporal (IMC), que se define como la relación entre el peso corporal dividido entre la talla elevada al cuadrado $\left(\mathrm{kg} / \mathrm{m}^{2}\right){ }^{(12)}$. La edad se clasificó 
en cuatro rangos: 15 - 20 años, 21 - 30 años, $31-40$ y >41 años. Se registraron también el peso pregestacional.

La edad gestacional se determinó "a partir del primer día del último periodo menstrual normal y el momento en que se realiza el cálculo, se expresa en días o semanas completas" (13), en nuestro caso, será dividida en primer trimestre (1-13 semanas), segundo (14-26 semanas) y tercero (27-41 semanas).

\section{Análisis estadístico}

Se empleó el programa estadístico SPSS versión 22.0 para Windows. Se definieron promedio y desviación estándar (DS) a las variables cuantitativas y se realizó estadística descriptiva de variables nominales con lo que se obtuvo frecuencia y porcentaje de las mismas. Los resultados se presentaron en tablas y se consideró el número total de pacientes con embarazo.

Para medir la variable de hemoglobina se realizaron pruebas estadísticas y se obtuvo como resultado que esta variable no sigue una distribución normal, por lo que se utilizó la prueba no paramétrica de Kolmogorov-Smirnov. Para la comparación de los niveles de hemoglobina, según las características sociodemográficas, se empleó la prueba
U de Mann-Whitney o la prueba de Kruskal-Wallis según análisis. Para la asociación entre la presencia de anemia y estas características se aplicó la prueba Chi-Cuadrado; en todos los casos se consideró significativo un valor $p<0$.

\section{Consideraciones éticas}

La realización del estudio requirió la aprobación de la Dirección de Salud II Lima Sur - Unidad de Capacitación de la Red de Barranco - Chorrillos - Surco.

\section{RESULTADOS}

La edad promedio de la población estudiada fue $24 \pm 5,8$ años, la edad gestacional media fue $14,0 \pm 7,5$ semanas; el peso, $59,5 \pm 11,9 \mathrm{~kg}$; la talla, $1,55 \pm 0,0 \mathrm{~m}$, y la hemoglobina promedio, $12,1 \pm 1,0 \mathrm{gr} / \mathrm{dl}$.

Noventa y tres pacientes cumplieron con los criterios de inclusión. El 20,2 \% tenía entre 17 - 20 años; 59,6\%, entre 21 y 30 años; $18,1 \%$, de 31 a 40 años; y 2,1\%, tenía más de 41 años. El 53,8 \% de las participantes se encontraban en su primera gestación. El 69,1\% de las embarazadas eran casadas/convivientes, y 65,6\% tenía secundaria completa. El $59,1 \%$ de las gestantes que acudieron a control se encontraban en el primer trimestre.

Tabla 1. Características generales de las gestantes atendidas en el C.S. SURCO

\begin{tabular}{|lcc|}
\hline EDAD (Años) & $\mathbf{n}$ & $\%$ \\
\hline $17-20$ & 19 & 20,4 \\
\hline $1-30$ & 55 & 59,2 \\
$31-40$ & 17 & 18,3 \\
$41-50$ & 2 & 2,1 \\
\hline EDAD GESTACIONAL (Trimestre) & $\mathbf{n}$ & $\%$ \\
\hline Primer (1- 13 semana) & 55 & 59,1 \\
\hline Segundo (14 - 26 semana) & 32 & 34,4 \\
\hline Tercer (27 - 40 semana) & 6 & 6,5 \\
\hline HEMOGLOBINA gr/dI & & \\
\hline Normal (>11) & $\mathbf{n}$ & $\%$ \\
\hline Anemia leve (9 - 11) & 82 & 88,2 \\
\hline Anemia moderada (7 -9) & 11 & 11,8 \\
\hline Anemia severa (<7) & 0 & 0,0 \\
\hline IMC PREGESTACIONAL & 0 & 0,0 \\
\hline Normal & $\mathbf{n}$ & $\%$ \\
\hline Sobrepeso & 55 & 59,1 \\
\hline Obesidad & 22 & 23,7 \\
\hline
\end{tabular}




\begin{tabular}{|lcc|}
\hline N. ${ }^{\circ}$ DE HIJOS & $\mathbf{n}$ & $\%$ \\
\hline 0 & 50 & 53,8 \\
$1-2$ & 32 & 34,4 \\
$3-4$ & 10 & 10,7 \\
$5-6$ & 1 & 1,1 \\
\hline GRADO DE INSTRUCCIÓN & $\mathbf{n}$ & $\%$ \\
\hline Primaria & 5 & 5,4 \\
\hline Secundaria & 61 & 65,6 \\
Superior universitario & 14 & 15,1 \\
\hline Superior no universitario & 8 & 8,6 \\
\hline Sin dato & 5 & 5,3 \\
\hline ESTADO CIVIL & $\mathbf{n}$ & $\%$ \\
\hline Casada /Conviviente & 64 & 68,8 \\
\hline Soltera & 29 & 31,2 \\
\hline
\end{tabular}

IMC: Índice de masa corporal

Fuente: Historia clínica materna perinatal.

El 11,8\% de gestantes tenían niveles bajos de hemoglobina, lo que las ubica dentro del diagnóstico de anemia leve, según la clasificación de la Organización Mundial de la Salud (OMS). No se encontraron casos de anemia moderada ni severa. El 5,3\% de las gestantes estaban en el grupo etario de 17-30 años, y 1,1\% en el de 31-40 años.

El promedio de $\mathrm{Hb}$ en relación a la edad fue de $11,8 \mathrm{~g} / \mathrm{dl}$ en el rango de 17 a 20 años; $12,3 \mathrm{~g} / \mathrm{dl}$, entre los 21 a 30 años; $12,2 \mathrm{~g} / \mathrm{dl}$, en el grupo de 31 a 40 años; y $12,5 \mathrm{~g} / \mathrm{dl}$, entre los 41-50 años.

En relación al inicio del control prenatal, 4,3 \% de las gestantes lo iniciaron en el primer trimestre, y 5,4\%, durante el segundo. El grupo que comenzó el control prenatal en tercer trimestre presentó $2,1 \%$ de casos de anemia. En total , se presenta un $11,8 \%$ de gestantes con anemia.

En este grupo comparativo observamos que la frecuencia de anemia es mayor cuando la gestante presenta un IMC pregestacional normal. Obtuvimos $1,8 \%$ de casos de anemia con un IMC de sobrepeso, mientras que no hallamos nivel de delgadez en ningún rango del IMC. Existió una diferencia significativa de los valores de hemoglobina según el estado nutricional (valor de $p<0,008$ ).

Tabla 2. Presencia de anemia en gestantes según índice de masa corporal (IMC) pregestacional en el Centro de Salud de Santiago de Surco

\begin{tabular}{lccccc|}
$\begin{array}{l}\text { Estado nutricional } \\
\text { pregestacional }\end{array}$ & Anémicas & No anémicas & p=valor \\
\hline Normal (n: 55$)$ & $\mathbf{N}^{\circ}$ & $\%$ & $\mathbf{N}^{\circ}$ & $\%$ & \\
Sobrepeso $(\mathrm{n}: 22)$ & 10 & 1,2 & 45 & 81,8 & $0.008^{*}$ \\
Obesidad (n: 16$)$ & 1 & 1,8 & 21 & 98,2 & \\
& 0 & 0 & 16 & 100,0 & \\
\end{tabular}

*Prueba de Kruskal- Wallis 


\section{Limitaciones}

Las historias clínicas de las que se obtuvieron las características generales de las gestantes atendidas en el C. S. Santiago de Surco estaban incompletas, lo que redujo la muestra a estudiar.

\section{DISCUSIÓN}

En el estudio realizado por Hernández-Vásquez et al. (14), la prevalencia nacional de anemia en gestantes fue de 24,2 \%, con mayor prevalencia en las regiones de Huancavelica, Puno, Pasco, Cusco y Apurímac; mientras que el mayor número de gestantes con anemia se concentró en las áreas de residencia rurales de las tres regiones naturales del Perú.

La anemia en gestantes atendidas en el Centro de Salud de Surco del Ministerio de Salud del Perú fue de 11,8 \%, un valor bajo, si se consideran las estimaciones realizadas por la OMS. La frecuencia de anemia global en gestantes reportada por Gonzales et al.(9); es cercana al 18,1 \%; mientras que en otros estudios se encontraron valores más altos como en el ENDES $2013(29,5 \%)^{(4)}$, Gonzales et al. $(18,1 \%)^{(9)}$, y Agreda et al. $(27,2 \%)^{(15)}$.

La edad de la muestra de gestantes en estudio fue de 25,4 $\pm 5,8$ años, resultado que coincide con la mediana del nacimiento del primer hijo de las mujeres en nuestro país que es 22 años, según la última Encuesta Demográfica y de Salud Familiar 2013 del Instituto Nacional de Estadística e Informática (INEI) ${ }^{(4)}$. Igualmente, en el estudio de Tapia (16) la edad encontrada fue 24,48 años; además, dicha encuesta reporta que en nuestro país el $64,6 \%$ de mujeres han tenido paridad a los 25 años.

Se identificó un 11,8 \% de anemia leve; $0 \%$ de anemia moderada y severa, cifras distintas al estudio de Munares et al., que identificó anemia leve entre $17,3 \%$ y $18,1 \%$; la anemia moderada, entre $9 \%$ y $9,7 \%$, y la de menor frecuencia fue la anemia severa entre $0,2 \%$ a $0,3 \%{ }^{(17)}$; Castillo encontró a 21 pacientes $(n=5)$ con anemia leve (46, 6\%), 19 con anemia moderada (42,2\%), y en el $11,1 \%$ de las pacientes $(n=5)$, se encontró anemia severa ${ }^{(18)}$. Se observa que la cifra en el presente estudio es menor para la anemia leve frente a los otros estudios.

A inicio del control prenatal, el 59,1\% de las gestantes se encontraban en el primer trimestre de gestación con niveles bajos de hemoglobina; Gómez-Sánchez et al. encontraron la misma situación en las gestantes que acudieron a su control en el primer trimestre ${ }^{(19)}$. La importancia del control prenatal en el primer trimestre es fundamental ya que el nivel de hemoglobina disminuye de manera significativa.

En cuanto a la prevalencia de casos de anemia en gestantes según el trimestre de gestación, se observó que es mayor en los dos primeros trimestres con un promedio de 4,8\%; mientras que en el tercer trimestre es menor con un 2,2 \%; datos similares a los de Vite $\mathrm{G}$ et al. que mencionan que el $15,3 \%$ de los casos de anemia se encuentran en el primer y segundo trimestre y luego se reducen en el tercer trimestre $(10,2 \%)$ (20). Por otro lado, Castillo et al. reportan que el $62,2 \%$ de las gestantes con anemia se encontraban en el tercer trimestre de embarazo ${ }^{(18)}$; Escudero et al. encontraron que la prevalencia es mayor conforme avanza la gestación (21); igualmente, San Gil Suárez et al. observaron una disminución de los valores medios de hemoglobina entre el primer trimestre $(11,2 \mathrm{~g} / \mathrm{dL})$ y el tercer trimestre $(10,8 \mathrm{~g} / \mathrm{dL}){ }^{(22)}$.

Los datos mencionados en el estudio de Munares-García et al. se muestra que los casos de anemia en el primer trimestre fueron del 15,3\%; en el segundo trimestre fue de $24,9 \%$ y en el tercer trimestre fue de $30,0 \%{ }^{(17)}$. Por lo tanto, es posible establecer que las carencias nutricionales figuren entre las causas principales de las anemias diagnosticadas, como lo reportado en el estudio de Becerra y González en el $2012{ }^{(23)}$.

Existe una relación entre la edad de la gestante y la anemia ferropénica. Esta patología se presentó en mayor proporción en el rango de 17- 30 años; mientras que en el rango de 31 años a más se encontró tan solo un 2,2 \% de casos de anemia; esta información se opone al estudio de Vite et al., en el que las edades de las gestantes anémicas oscilaban entre los 19 a 43 años ${ }^{(20)}$.Como observamos, los niveles de hemoglobina disminuyen de acuerdo a la edad de la madre, esto se debería a un factor de malnutrición y a cambios fisiológicos.

Con respecto a la frecuencia de anemia según IMC pregestacional, se pudo determinar que las gestantes con sobrepeso tienen menor frecuencia de anemia $(1,1 \%)$ que las que se encuentran con IMC pregestacional normal $(10,7 \%)$. Datos similares se ven reflejados en el estudio de Munares-García et al., en el que las gestantes añosas obesas presentaron menor número de casos de anemia $(21,9 \%)$ que las normales, en quienes se observa mayor número de casos de anemia $(30,7 \%){ }^{(17)}$. En contraste con nuestros resultados, en el estudio de Becerra et al. no se encontraron diferencias entre el peso de la madre al inicio del embarazo y la gravedad de su anemia gestacional (23). Se sabe que durante la gestación los niveles de hemoglobina tienden a reducirse y esto es marcado en los casos en que se tienen pocas reservas de hierro, puesto que la reducción de los niveles de hemoglobina es un indicador tardío de las reservas de hierro ${ }^{(8)}$.

En conclusión, la frecuencia de anemia en las gestantes del Centro de Salud de Surco fue de 11,8 \% de anemia leve, no encontramos casos de anemia moderada o severa. No hay relación entre la presencia de IMC pregestacional 
bajo y anemia. Los niveles de hemoglobina disminuyen conforme a la edad de la madre, y aumentan de acuerdo a la edad gestacional.

Agradecimiento: A la Dra. Luzmila Troncoso Corzo, Lic. Fernando Flores Escudero por su apoyo técnico en la investigación realizada.

Contribución de autoría: Blanca Regina Taipe Ruiz que ha realizado diseño del estudio, recolección e interpretación de los datos y elaboración del informe final de investigación. Luzmila Troncoso Corzo ha realizado diseño del estudio, interpretación de los datos, revisión del informe final de la investigación.

\section{REFERENCIAS BIBLIOGRÁFICAS}

1. Directiva Sanitaria para la prevención de anemia mediante la suplementación con micronutrientes y hierro en niñas y niños menores de 36 meses de edad. Resolución Ministerial $\mathrm{N}^{\circ}$ 0552016. Lima: Ministerio de Salud. Disponible en: http://bvs. minsa.gob.pe/local/MINSA/3931.pdf

2. Instituto Nacional de Estadística e Informática. Encuesta Demográfica y de Salud Familiar - ENDES 2012. [Internet]. Lima, Perú: INEI; 2012. Disponible en: http://www.inei. gob. pe/srienaho/Consulta_por_Encuesta.asp

3. Gómez-Guizado G, Barzola-Del Carpio CJ, Munares-García O, Prado-Merino F, Cosser-Gamarra C. Vigilancia del estado nutricional en niños y gestantes - I semestre 2014. Bol - Inst Nac Salud 2014; 20 (7-8) julio - agosto.

4. Instituto Nacional de Estadística e Informática. Encuesta Demográfica y de Salud Familiar - ENDES 2013. [Internet]. Lima: INEI; 2013. Disponible en: http://www.inei. gob.pe/ srienaho/Consulta_por_Encuesta.asp

5. Mendoza L, Pérez B, Sánchez S. Estado nutricional de embarazadas en el último mes de gestación y su asociación con las medidas antropométricas de sus recién nacidos. Pediatr (Asunción). 2010; 37(2): 91-6.

6. Directiva Sanitaria para la Prevención y Control de la Anemia por Deficiencia de Hierro en Gestantes y Puérperas. Resolución Ministerial $N^{\circ}$ 069-2015. Lima: Ministerio de Salud. Disponibleen: http: //200.37.75.52/portal/wp-content/uploads/2018/02/ CONSEJER\%C3\%8DA-EN-SUPLEMENTACI\%C3\%93N.pdf

7. Reveiz L, Gyte GML, Cuervo LG. Tratamientos para la anemia ferropénica en el embarazo. Base de Datos de Revisiones Sistemáticas. En: La Biblioteca Cochrane Plus, número 2, 2001. Oxford, Update Software Ltd.

8. Palacios-Solano J, Peña-Ayudante W. Prevalencia de anemia en gestantes de la ciudad de Huacho. Rev Soc Peru Med Interna. 2014; 27(1): 6-11.

9. Gonzales G, Gonzales C. Hierro, anemia y eritrocitosis en gestantes de la altura: riesgo en la madre y el recién nacido. Rev Perú Ginecol Obstet. 2012; 58(4): 329-340.

10. Norma Técnica: Manejo Terapéutico y Preventivo de la anemia en niños, adolescentes, mujeres gestantes y puérperas. Lima: Ministerio de Salud, 2016. Disponible en: http://bvs.minsa. gob.pe/local/MINSA/4190.pdf

11. Instituto Nacional de Salud. Centro Nacional de Alimentación y Nutrición. La Medición de la Talla y el Guía para el personal de salud del primer nivel de atención. Lima: INS; 2004. Disponible en: https://repositorio.ins.gob.pe/handle/INS/219

12. Gonzales G, Tapia V, Gasco M, Carrillo C. Hemoglobina materna en el Perú: diferencias regionales y su asociación con resultados adversos perinatales. Rev Perú Med Exp Salud Pública. 2011; 28(3):484-91.
13. Guía técnica: Consejería nutricional en el marco de la atención integral de salud de la gestante y puérpera. Lima: Ministerio de Salud, 2016. Disponible en: https://web. ins.gob.pe/sites/default/files/Archivos/cenan/deprydan/ documentosNormativos/2_Guia_Gestante_final-ISBN.pdf

14. Hernández-Vásquez A, Azañedo D, Antiporta D, Cortés S. Análisis espacial de la anemia gestacional en el Perú, 2015. Rev Perú Med Exp Salud Pública. 2017; 34 (1): 43-51.

15. Agreda L, Rosales S, Alarcón-Matutti E, Gutiérrez C, GómezSánchez I,Castillo A. Nivel de hemoglobina y prevalencia de anemia en gestantes según características socio-demográficas y prenatales. Rev Perú Epidemiol. 2014; 18(2):1-6

16. Miranda Tapia AM. Anemia en gestantes y peso del recién nacido. Hospital Nacional Arzobispo Loayza 2014. [Tesis de Título de Especialista en Ginecología y Obstetricia]. Lima: Universidad San Martín de Porres. 2015:61pp

17. Munares-García O, Gómez-Guizado G, Anemia en gestantes añosas atendidas en los establecimientos del Ministerio de Salud del Perú, 2009-2012. Rev Perú Epidemiol. 2016; 18(2): 1-7.

18. Ayala Castillo RF. Prevalencia de anemia en gestantes que acuden por signos de alarma al servicio de emergencia del Hospital Nacional Arzobispo Loayza del 1 junio al 30 de noviembre de 2016. [Tesis de Médico Cirujano]. Lima: Universidad Nacional Mayor de San Marcos. 2017:53pp

19. Gómez-Sánchez I, Rosales S, Agreda L, Castillo A, AlarcónMatutti E, Gutiérrez C. Nivel de hemoglobina y prevalencia de anemia en gestantes según características socio-demográficas y prenatales. Rev Perú Epidemiol. 2014; 18(2): 1-6.

20. Vite F. Incidencia de anemia ferropénica y factores asociados en las gestantes del distrito de Rapayan, Ancash, Perú: Período mayo 2010-marzo 2011. Acta Méd Peruana. 2011;28(4): 184-7.

21. Escudero L, Parra E, Restrepo S. Factores sociodemográficos y gestacionales asociados a la concentración de hemoglobina en embarazadas de la Red Hospitalaria Pública de Medellín. Rev Chil Nutr. 2011; 38(4): 429-37.

22. San Gil C, Villazán C, Ortega Y. Caracterización de la anemia durante el embarazo y algunos factores de riesgo asociados, en gestantes del municipio regla. Rev Cubana Med Gen Integr. 2014; 30(1): 71-81.

23. Becerra C, González F. Villena A, De la Cruz D, Florián A. Prevalencia de anemia en gestantes Hospital Regional Pucallpa Perú. Rev Panam Salud Pública. 1998; 3(5):285-92.

Fuentes de financiamiento:

Este artículo ha sido financiado por los autores.

Conflictos de interés:

Los autores declaran no tener conflictos de interés.

Correspondencia:

Blanca Regina Taipe Ruiz

Dirección: Urb. Virgen del Carmen Mz B Lt 22, Ate Vitarte. Lima, Perú. Teléfono: 987953148

Correo electrónico: blanca0906@hotmail.com

Recibido: 13 de agosto de 2018. Evaluado: 09 de octubre de 2018. Aprobado: 12 de noviembre 2018.

(c) La revista. Publicado por Universidad de San Martín de Porres, Perú. (c) ${ }^{\text {в }}$ Licencia de Creative Commons Artículo en acceso abierto bajo términos de Licencia Creative Commons Atribución 4.0 Internacional. (http://creativecommons.org/licenses/by/4.0/)

\section{ORCID iDs}

Blanca Regina Taipe Ruiz Luzmila Troncoso Corzo

https://orcid.org/0000-0002-0345-6922 https://orcid.org/0000-0003-1075-874X 